\title{
Pulmonary intravascular large B-cell lymphoma successfully treated with rituximab, cyclophosphamide, vincristine, doxorubicin and prednisolone immunochemotherapy: Report of a patient surviving for over 1 year
}

\author{
SHIZUKA NISHII-ITO ${ }^{1,2}$, HIROKI IZUMI ${ }^{2}$, HIROKAZU TOUGE ${ }^{2}$, KENICHI TAKEDA $^{2}$, \\ YUZURU HOSODA $^{1,3}$, AKIRA YAMASAKI ${ }^{2}$, SATOSHI KUWAMOTO ${ }^{4}$, EIJI SHIMIZU ${ }^{2}$ and TORU MOTOKURA ${ }^{1,3}$ \\ ${ }^{1}$ Department of Hematology, Tottori University Hospital, Yonago, Tottori 683-8504; \\ ${ }^{2}$ Division of Medical Oncology and Molecular Respirology, Department of Multidisciplinary Internal Medicine; \\ ${ }^{3}$ Division of Clinical Laboratory Medicine, Department of Pathophysiological and Therapeutic Science; \\ ${ }^{4}$ Division of Molecular Pathology, Department of Pathology, Faculty of Medicine, \\ Tottori University, Yonago, Tottori 683-8503, Japan
}

Received July 14, 2016; Accepted September 5, 2016

DOI: $10.3892 / \mathrm{mco} .2016 .1063$

\begin{abstract}
A 73-year-old man with a history of lethargy, fever and dyspnea was admitted to Tottori University Hospital. A computed tomography (CT) scan revealed splenomegaly and diffusely spreading ground-glass opacities (GGOs) in both lungs. A video-assisted thoracoscopic surgery (VATS)-guided lung biopsy revealed intravascular proliferation of large atypical lymphoid cells in the arteries, veins and alveolar walls. The patient was diagnosed with intravascular large B-cell lymphoma (IVLBCL); he received 6 cycles of rituximab, cyclophosphamide, doxorubicin, vincristine and prednisolone (R-CHOP) immunochemotherapy and has remained in complete remission for $>1$ year. Although IVLBCL is a rare disease, it should be considered in the differential diagnosis of pulmonary diffuse lesions that present with GGOs on CT scans.
\end{abstract}

\section{Introduction}

Intravascular large B-cell lymphoma (IVLBCL) is a rare entity characterized by exclusive or predominant growth of neoplastic cells within the lumen of blood vessels. This disease was recently recognized as a subtype of diffuse large B-cell lymphoma by the World Health Organization (WHO) (1). The clinical presentation of IVLBCL varies widely due to

Correspondence to: Professor Toru Motokura, Division of Clinical Laboratory Medicine, Department of Pathophysiological and Therapeutic Science, Faculty of Medicine, Tottori University, 86 Nishi-cho, Yonago, Tottori 683-8503, Japan

E-mail: motokura@med.tottori-u.ac.jp

Key words: intravascular large B-cell lymphoma, rituximab, ground-glass opacities occlusion of small vessels or capillaries in different organs. The most common clinical symptoms include fever, general fatigue, neurological manifestations and non-specific skin eruptions $(2,3)$. Although autopsy findings have revealed that pulmonary involvement is also common in this disease, a primary presentation in the lung is uncommon and has rarely been described. In non-Hodgkin lymphoma (NHL), extranodal disease accounts for $24-50 \%$ of all cases, whereas only $34 \%$ of the cases include pulmonary lesions, with primary pulmonary lesions presenting in $<1 \%$ of the patients (4). As early pulmonary IVLBCL diagnosis is difficult and treatment for pulmonary IVLBCL is less effective, the prognosis for the majority of the patients is poor (4). We herein report the case of a patient with pulmonary IVLBCL who was successfully treated with R-CHOP and has survived for $>1$ year.

\section{Case report}

A 73-year-old man visited a local clinic complaining of general malaise, dry cough, hoarseness, a 6-kg weight loss and fever. The patient was referred to our hospital, as no improvement was observed after 3 months. The patient's medical history was unremarkable, he had never smoked and had no history of occupational dust exposure. On admission, there were no enlarged superficial lymph nodes, skin lesions, or neurological abnormalities There were no abnormal findings on chest auscultation. Laboratory tests revealed the following results: Total leukocyte count, 5,400 cells $/ \mathrm{mm}^{3}$ (69\% neutrophils, $11 \%$ lymphocytes, $20 \%$ monocytes, $0 \%$ basophils and eosinophils); hemoglobin concentration, $11.6 \mathrm{~g} / \mathrm{dl}$; mean corpuscular volume, $82.5 \mathrm{fl}$; reticulocyte percentage, $8 \%$; platelet count, 117,000/ $\mu 1$; C-reactive protein, $17.9 \mathrm{mg} / \mathrm{dl}$; ferritin, $5,720 \mathrm{ng} / \mathrm{ml}$; serum lactate dehydrogenase, $562 \mathrm{U} / 1$; and soluble interleukin-2 receptor, 8,313 U/ml. Hypoxemia was also present, with an arterial oxygen tension of 53.2 Torr (room air). The pulmonary function test results were within 

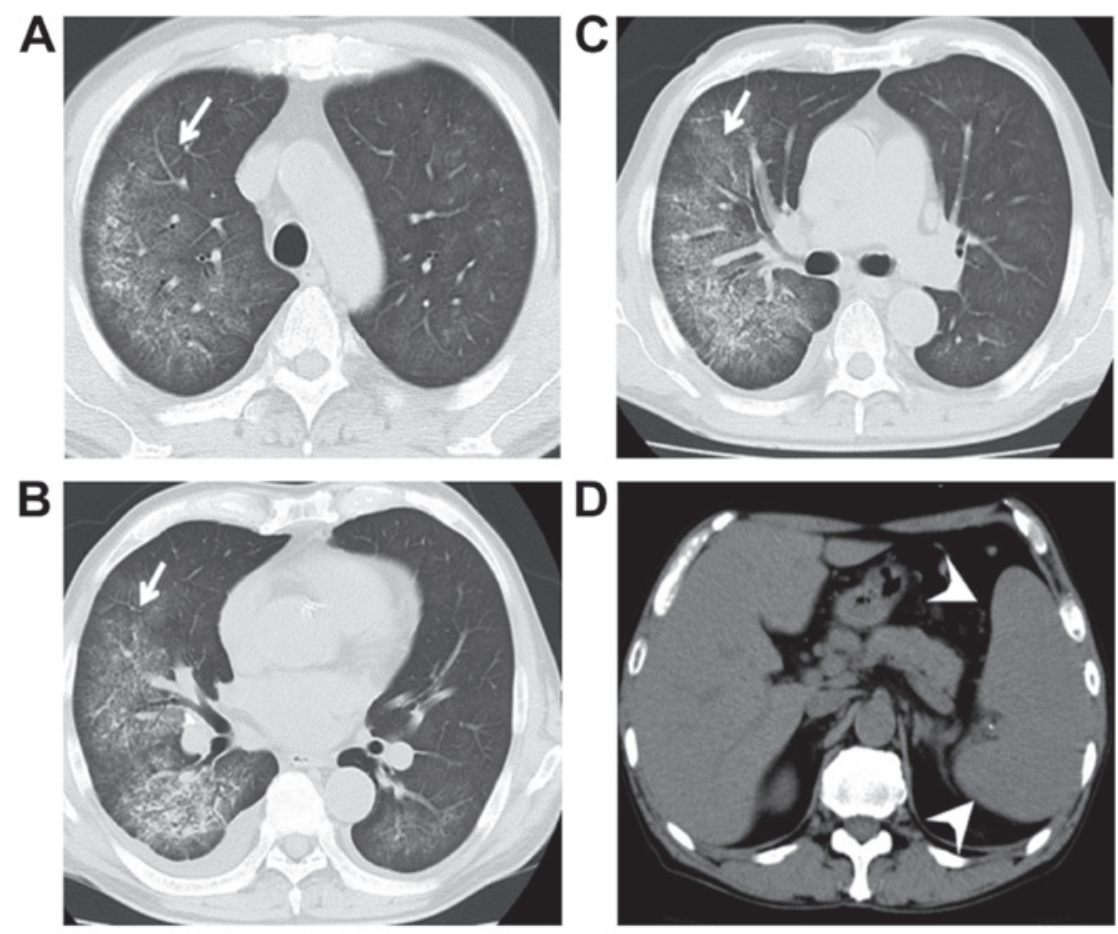

Figure 1. Chest computed tomography showing (A-C) multiple ground glass opacities (arrows) in both lungs and (D) splenomegaly (arrowheads).
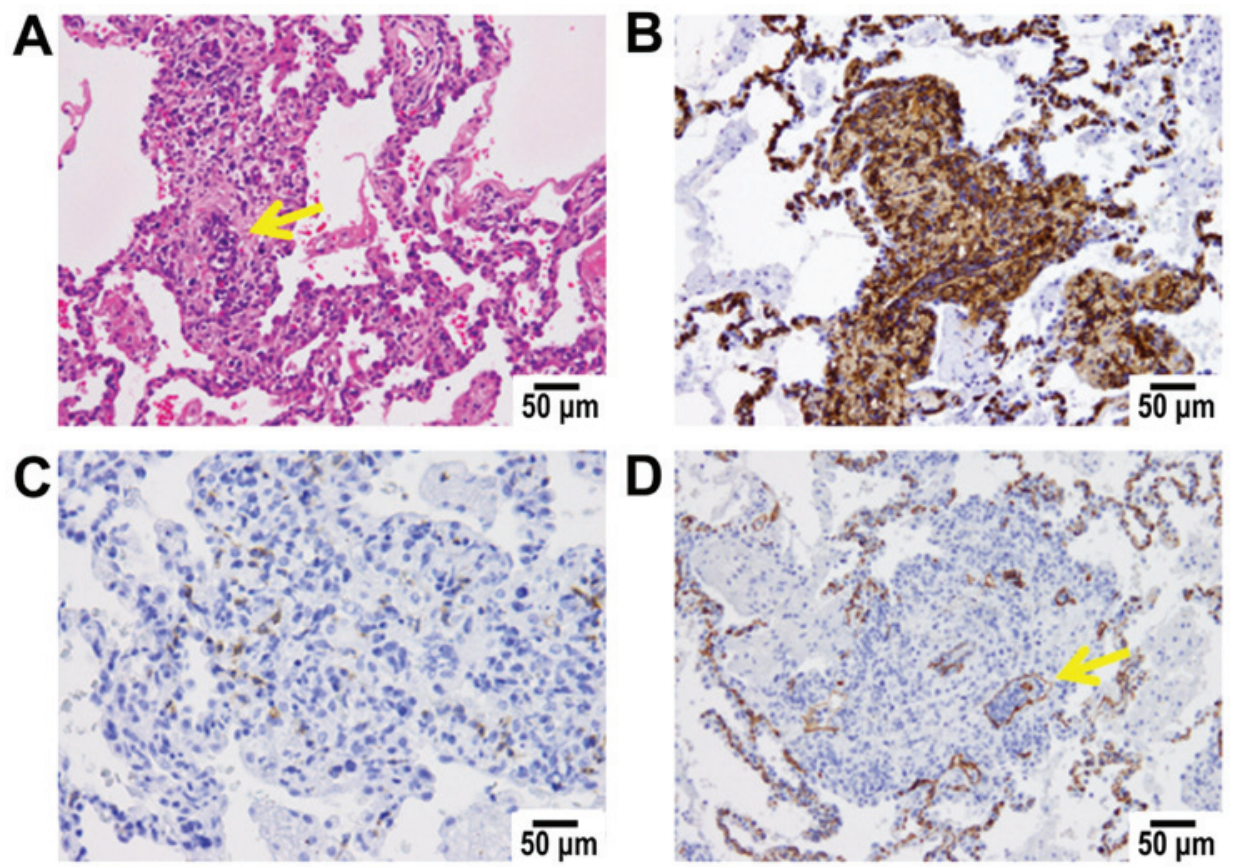

Figure 2. Histological diagnosis of intravascular large B-cell lymphoma. (A) Hematoxylin and eosin staining showing several large atypical cells (arrow). (B) CD20 immunostaining of atypical cells. (C) Negative CD5 immunostaining (D) CD34 immunostaining showing atypical cells mainly located within small vessels and alveolar wall capillaries (arrow).

normal limits, except for a reduction in the diffusion capacity of carbon monoxide (64.3\% of the predicted value). There were no laboratory data suggestive of infection or autoimmune disease.

Chest radiography revealed diffusely spreading ground-glass opacities (GGOs) in both lungs, predominantly in the inferior lobes, but there were no signs of cardiac enlargement, pleural effusion, or enlarged mediastinal lymph nodes. Bilateral diffuse GGOs were also evident on computed tomography (CT) scans, but there were no signs of interlobular septal thickening or decreased volume. The spleen was also enlarged on CT (Fig. 1). Gallium scintigraphy also revealed diffuse and increased uptake in the lung field, as well as increased uptake in the spleen and splenomegaly. 

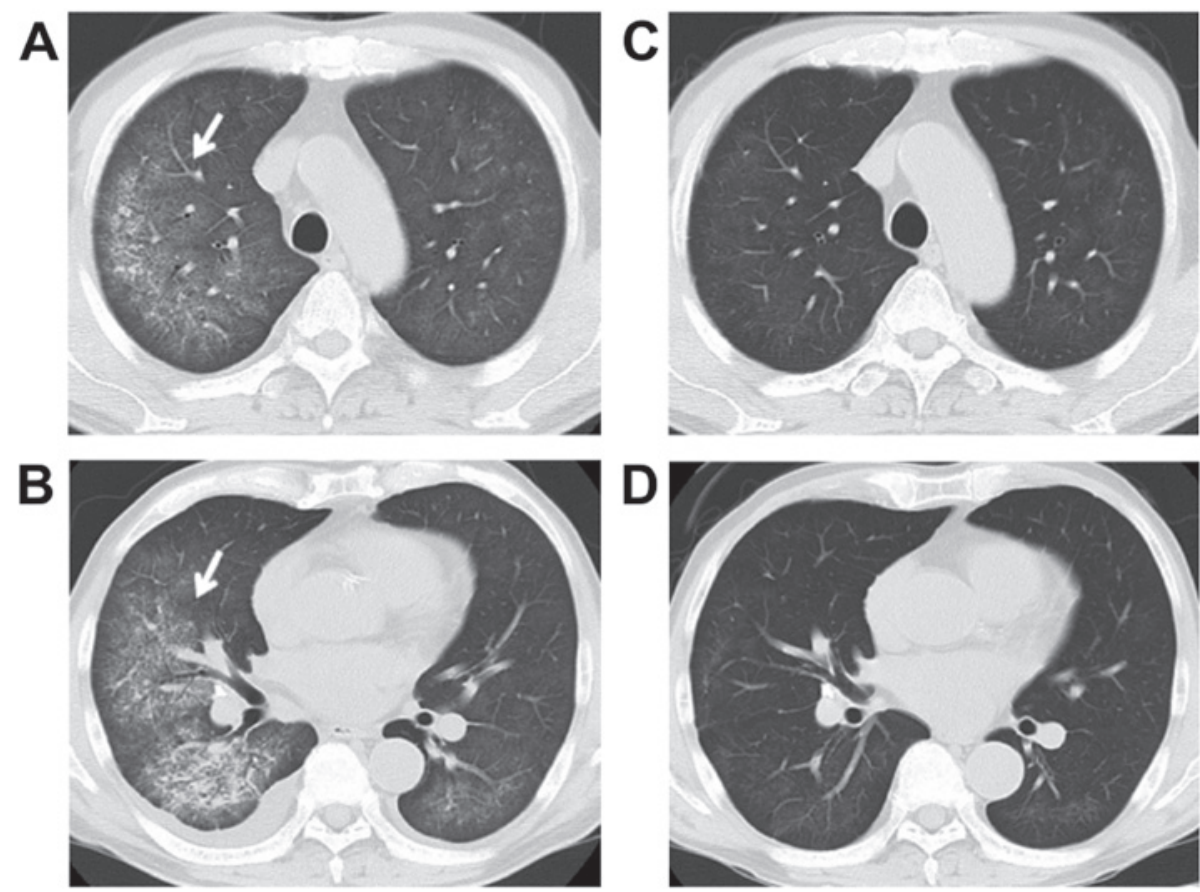

Figure 3. Repeat chest computed tomography showing resolution of the multiple ground glass opacities (GGOs) in the lungs (arrows). (A and B) Multiple GGOs in both lungs were observed upon admission to the hospital. (C and D) The GGOs rapidly disappeared after treatment.

At that point, the differential diagnosis included atypical pneumonia, eosinophilic pneumonia, hypersensitivity pneumonitis, pulmonary alveolar hemorrhage, pulmonary alveolar proteinosis and intravascular large B-cell lymphoma. However, a transbronchial lung biopsy (TBLB) could not be performed due to the patient's coughing. Antibiotic and steroid treatment was initiated, but the patient's general condition continued to deteriorate. A lung biopsy was then performed using video-assisted thoracoscopic surgery (VATS).

Histological examination of the bioptic specimen revealed several large atypical cells, mainly located within the small vessels and alveolar wall capillaries (Fig. 2A and D). These atypical cells also infiltrated an extravascular area of the stroma, with a patchy distribution in the enlarged interstitium throughout the lesion. Imunohistochemically, the atypical cells were positive for CD20 (Fig. 2B) but were negative for CD5 (Fig. 2C). Epstein-Barr virus (EBV)-encoded RNA in situ hybridization was also performed, but only a few positive cells were detected, ruling out involvement of EBV in this case. Bone marrow infiltration was not observed, although phagocytosis of neutrophils by macrophages was present at a very low frequency. The findings of the skin biopsy were unremarkable. Based on these findings, the patient was diagnosed with IVLBCL.

As respiratory failure was progressing rapidly, steroid pulse therapy and oral cyclophosphamide (100 mg per day for 6 days) were administered until the histological results became available. After diagnosis was confirmed, six courses of R-CHOP immunochemotherapy were administered. The GGOs in the lung field rapidly disappeared and the respiratory status improved (Fig. 3). A positron emission tomography (PET)/CT scan after the completion of the sixth course revealed no uptake in the lung or spleen and the patient remains alive and symptom-free for $>1$ year.
The patient has provided his consent regarding the publication of the case details.

\section{Discussion}

IVLBCL is characterized by proliferation of atypical lymphoid cells within the lumen of capillaries. IVLBCL is a rare variant of diffuse large B-cell lymphoma that has been included in the WHO classification (5). The central nervous system (CNS) and skin are usually involved. By contrast, IVLBCL patients in Asian countries often exhibit hepatosplenomegaly, thrombocytopenia and fever, but CNS and skin involvement are rare $(6,7)$. In the present case, the patient exhibited findings typical of an Asian patient. In addition, several pulmonary IVLBCL cases were recently reported in Asia (2-4).

Recently, fluorodeoxyglucose (FDG)-PET/CT was shown to be useful for staging NHL (8), and several authors reported that FDG-PET/CT is a powerful tool for early diagnosis of IVLBCL (9); however, biopsy is essential. Due to its low invasiveness, TBLB is one of the most useful biopsy techniques for pulmonary lesions (4). In the present case, VATS-guided surgical biopsy was crucial, as TBLB could not be performed. If diffuse GGOs are present, IVLBCL should be included in the differential diagnosis, and a biopsy should be proactively performed.

The recommended treatment is systemic chemotherapy with an anthracycline-based regimen. However, the majority of IVLBCL cases are associated with poor prognosis. Recently, R-CHOP immunochemotherapy was shown to yield better results compared with $\mathrm{CHOP}(4,10,11)$. Our patient was treated with R-CHOP, and 1 year later there has been no recurrence. Pulmonary IVLBCL presenting with GGOs is exceedingly rare, but it should be considered in the differential diagnosis of respiratory disease presenting with GGOs. 


\section{Acknowledgements}

The authors are deeply grateful to Hiroshige Nakamura and Tomohiro Haruki who performed the biopsy using VATS. We are also indebted to Shu Nakamoto for their invaluable comments.

\section{References}

1. Swerdllow S, Campo E and Harris NL: WHO classification of tumours of haematopoietic and lymphoid tissues, IARC press, France, 2008.

2. Xiao D, Fu C, Long X, Liu W, Chen C, Zhou J and Fan S: Lung intravascular large B-cell lymphoma with ground glass opacities on chest computed tomography: A case report. Int J Clin Exp Pathol 7: 5285-5290, 2014.

3. Liu C, Lai N, Zhou Y, Li S, Chen R and Zhang N: Intravascular large B-cell lymphoma confirmed by lung biopsy. Int J Clin Exp Pathol 7: 6301-6306, 2014.

4. Chen Y, Ding C, Lin Q, Yang K, Li Y and Chen S: Primary intravascular large B-cell lymphoma of the lung: A review and case report. J Thorac Dis 6: E242-E245, 2014.

5. Kotake T, Kosugi S, Takimoto T, Nakata S, Shiga J, Nagate Y, Nakagawa T, Take H and Katagiri S: Intravascular large B-cell lymphoma presenting pulmonary arterial hypertension as an initial manifestation. Intern Med 49: 51-54, 2010.

6. Kawamura T, Sando Y, Tajima S, Hosono T, Sato M, Maeno Y, Maeno T, Suga T, Kurabayashi M and Nagai R: Pulmonary intravascular lymphoma complicated with Pneumocystis carini pneumonia: A case report. Jpn J Clin Oncol 31: 333-336, 2001.

7. Takamura K, Nasuhara Y, Mishina T, Matsuda T, Nishimura M Kawakami Y, Fujita M, Mikuni C and Yamashiro K: Intravascular lymphomatosis diagnosed by transbronchial lung biopsy. Eur Respir J 10: 955-957, 1997.
8. Juweid ME, Stroobants S, Hoekstra OS, Mottaghy FM, Dietlein M, Guermazi A, Wiseman GA, Kostakoglu L, Scheidhauer K, Buck A, et al: Use of positron emission tomography for response assessment of lymphoma: Consensus of the imaging subcommittee of international harmonization project in lymphoma. J Clin Oncol 25: 571-578, 2007.

9. Yamashita H, Suzuki A, Takahashi Y, Kubota K, Kano T and Mimori A: Intravascular large B-cell lymphoma with diffuse FDG uptake in the lung by 18FDG-PET/CT without chest CT findings. Ann Nucl Med 26: 515-521, 2012.

10. Zinzani PL, Martelli M, Poletti V, Vitolo U, Gobbi PG, Chisesi T, Barosi G, Ferreri AJ, Marchetti M, Pimpinelli N, et al: Practice guidelines for the management of extranodal non-Hodgkin's lymphomas of adult non-immunodeficient patients. Part I: Primary lung and mediastinal lymphomas. A project of the Italian society of hematology, the Italian society of experimental hematology and the Italian group for bone marrow transplantation. Haematologica 93: 1364-1371, 2008

11. Murase T, Yamaguchi M, Suzuki R, Okamoto M, Sato Y, Tamaru J, Kojima M, Miura I, Mori N, Yoshino T and Nakamura S: Intravascular large B-cell lymphoma (IVLBCL): A clinicopathologic study of 96 cases with special reference to the immunophenotypic heterogeneity of CD5. Blood 109: 478-485, 2007. 